\title{
Cluster molecular-orbital calculations on germanium adsorbed on $\mathrm{Si}(111)$ surfaces
}

\author{
Michael Grodzicki* and Marcus Wagner ${ }^{\dagger}$ \\ Hamburger Synchrotronstrahlungslabor (HASYLAB) at Deutsches Elektronen-Synchrotron (DESY), Notkestrasse 85, \\ D-2000 Hamburg 52, West Germany
}

(Received 21 December 1988)

\begin{abstract}
Cluster model calculations on germanium adsorption onto the $\mathrm{Si}(111)-7 \times 7$ surface are performed with the self-consistent charge $X \alpha$ (SCC- $X \alpha$ ) method. Calculations on small silicon clusters and on the bare substrate prove the model's reliability for the subsequent investigations comprising adsorption of a single germanium atom on two different sites in the dimer-adatom-stacking-fault model, as well as that of two germanium atoms simultaneously. Adsorption energies, distances, and strengths of the respective $\mathrm{Si}-\mathrm{Ge}$ bonds are compared. Eventually, an explanation for the first steps of an interface formation between $\mathrm{Si}$ and $\mathrm{Ge}$ is offered.
\end{abstract}

\section{INTRODUCTION}

The structure of the Si(111) surface including adsorption processes is a subject of central interest. Nevertheless, sufficient theoretical information is still missing from the literature to interpret experimental data unambiguously and to investigate physical properties not amenable to experimental observation. In this paper, we present the first calculation of $\mathrm{Ge}$ adsorption on a $\mathrm{Si}(111)-7 \times 7$ surface to provide some insight into the initial steps of Ge layer growth on silicon reconstructed in the dimer-adatom-stacking-fault (DAS) model of Takayanagi et al. ${ }^{1,2}$

The interaction between a silicon substrate and germanium adsorbates is treated in the framework of the cluster model constituting an efficient procedure to handle such computations. The analysis is performed with the self-consistent charge $X \alpha(\mathrm{SCC}-X \alpha)$ method $^{3}$ which is a semiempirical procedure for calculating the electronic structure of large quantum-mechanical systems. This method has already been successfully applied to problems such as the adsorption of water dimers on nickel, ${ }^{4,5}$ the adsorption of various molecules in zeolites, ${ }^{6-8}$ and the reaction of $\mathrm{CO}$ with an imperfect $\mathrm{TiO}_{2}$ surface. ${ }^{9,10}$ In Sec. II, calculations on small silicon clusters are reported for the comparison of the SCC- $X \alpha$ method with $a b$ initio investigations. In Sec. III, we discuss the results of our cluster calculations on the bare $\mathrm{Si}(111)-7 \times 7$ surface, followed by the treatment of the Ge adsorption on this substrate in Sec. IV, and a comparison of the results with the experiment in Sec. V. We conclude with a brief summary and an outlook on future perspectives and projects.

\section{CALCULATIONS ON SMALL SILICON CLUSTERS}

In this section we discuss briefly our calculations on the clusters $\mathrm{Si}_{4}$ and $\mathrm{Si}_{5}$, in some geometries resembling the silicon bulk coordination. This permits a comparison of the SCC- $X \alpha$ method $^{3}$ with other procedures as well as an estimate of the reliability of our results when applied to modeling the $\mathrm{Si}(111)-7 \times 7$ surface. It should be point- ed out that no experimental information on the silicon clusters dealt with in this work has yet been published. Our numerical results are summarized in Table $\mathrm{I}$.

The cluster $\mathrm{Si}_{4}$ is computed for the two geometries corresponding to symmetries $D_{4 h}$ (square) and $T_{d}$ (tetrahedron), respectively. For $\mathrm{Si}_{4}\left(D_{4 h}\right)$ we obtain the same valence-electron configuration ${ }^{3} A_{2 g},\left(1 a_{1 g}\right)^{2}\left(1 e_{u}\right)^{4}$ $\left(1 b_{1 g}\right)^{2}\left(1 b_{2 g}\right)^{2}\left(1 a_{2 u}\right)^{2}\left(2 a_{1 g}\right)^{2}\left(2 e_{u}\right)^{2}$, as Raghavachari. ${ }^{11}$ from $a b$ initio Hartree-Fock calculations and as Pacchioni and Koutecky ${ }^{12}$ (PK) from an $a b$ initio procedure including some configuration interaction (the latter authors obtained a result with the second- and third-highest levels interchanged). For $T_{d}$ symmetry our configuration ${ }^{1} A_{1},\left(1 a_{1}\right)^{2}\left(1 t_{2}\right)^{6}\left(2 a_{1}\right)^{2}\left(2 t_{2}\right)^{6}$, is again identical with that of Raghavachari ${ }^{11}$ while PK (Ref. 12) compute, as the ground state, a triplet configuration, which is however, lower in energy by only $0.003 \mathrm{eV}$ than the ${ }^{1} A_{1}$ configuration. Our bond lengths for $\mathrm{Si}_{4}$ in $D_{4 h}$ and $T_{d}$ symmetry of 2.198 and $2.342 \AA$, respectively, are slightly shorter than those of Raghavachari ${ }^{11}$ (2.316 and 2.458 $\AA$ ). The bond lengths computed by PK (Ref. 12) seem to be unreasonably large at 2.47 and $2.71 \AA$, respectively. Comparing the two symmetries with each other we obtain the result that the $D_{4 h}$ is distinctly more stable, in qualitative agreement with Raghavachari ${ }^{11}$ and PK (Ref. 12) but in contrast to an older Hückel calculation of Martin and Schaber. ${ }^{13}$ Our result is supported by the fact that the overlap population of $0.886 e$ between two adjacent $\mathrm{Si}$ atoms in the $D_{4 h}$ geometry indicates a considerably stronger covalent bond than in $T_{d}$ geometry with $(0.453 e)$. This is easily understood because the bonds in the tetrahedron are bent and are energetically less favorable than the unstrained $(p p \sigma)$ bonds in the $D_{4 h}$ geometry.

$\mathrm{Si}_{5}$ (cf. Fig. 1) is computed for three geometries corresponding to the symmetries $D_{3 h}$ (trigonal bipyramid), $C_{4 v}$ (square pyramid), and $T_{d}$ (tetrahedron). According to our results, the trigonal bipyramid has the closed-shell valence-electron configuration ${ }^{1} A_{1}^{\prime}, \quad\left(1 a_{2}^{\prime}\right)^{2}\left(1 a_{2}^{\prime \prime}\right)^{2}\left(1 e^{\prime}\right)^{4}$ $\left(2 a_{1}^{\prime}\right)^{2}\left(1 e^{\prime \prime}\right)^{4}\left(3 a_{1}^{\prime}\right)^{2}\left(2 e^{\prime}\right)^{4}$, with a lateral Si-Si distance of $2.991 \AA$ and $a$ bond length between lateral and axial 
TABLE I. Ground state and bond length of small silicon clusters.

\begin{tabular}{|c|c|c|c|c|c|c|}
\hline \multicolumn{3}{|c|}{ Pacchioni and Koutecky (Ref. 12) } & \multicolumn{2}{|c|}{ Raghavachari (Ref. 11) } & \multicolumn{2}{|c|}{ Present work } \\
\hline Cluster & State & Bond length $(\AA)$ & State & Bond length (Å) & State & Bond length $(\AA)$ \\
\hline $\mathrm{Si}_{4}\left(D_{4 h}\right)$ & ${ }^{3} A_{2 g}$ & $d=2.47$ & ${ }^{3} A_{2 g}$ & $d=2.316$ & ${ }^{3} A_{2 g}$ & $d=2.198$ \\
\hline $\mathrm{Si}_{4}\left(T_{d}\right)$ & ${ }^{3} T_{2}$ & $d=2.71$ & ${ }^{1} A_{1}$ & $d=2.458$ & ${ }^{1} A_{1}$ & $d=2.342$ \\
\hline $\mathrm{Si}_{5}\left(D_{3 h}\right)$ & ${ }^{3} A_{2}^{\prime}$ & $d=2.63$ & ${ }^{1} A_{1}^{\prime}$ & $\begin{array}{l}d_{\text {lat-lat }}=3.256 \\
d_{\text {lat-ax }}=2.338\end{array}$ & ${ }^{1} A_{1}^{\prime}$ & $\begin{aligned} d_{\text {lat-lat }} & =2.991 \\
d_{\text {lat-ax }} & =2.342\end{aligned}$ \\
\hline $\mathrm{Si}_{5}\left(C_{4 v}\right)$ & ${ }^{1} A_{1}$ & $d=2.62$ & ${ }^{1} A_{1}$ & $\begin{aligned} d_{\text {lat-lat }} & =2.295 \\
d_{\text {lat-ax }} & =2.502\end{aligned}$ & ${ }^{1} A_{1}$ & $\begin{aligned} d_{\text {lat-lat }} & =2.306 \\
d_{\text {lat-ax }} & =2.491\end{aligned}$ \\
\hline $\mathrm{Si}_{5}\left(T_{d}\right)$ & ${ }^{5} E$ & $d=2.50$ & no & values published & ${ }^{1} A_{1 g}$ & $d=2.171$ \\
\hline
\end{tabular}

atoms of $2.342 \AA$. The respective bond lengths of Raghavachari $^{11}$ are 3.256 and $2.338 \AA$. The bonding results from only the lateral-axial bonds, whereas the small negative overlap populations between two lateral atoms $(-0.02 e)$ and between the two axial atoms $(-0.06 e)$ exhibit a weak repulsion. The calculation of PK (Ref. 12) may be unreliable insofar as they assume equal nextneighbor distance of $2.63 \AA$ for the lateral-lateral and the lateral-axial bonds. This is most likely the reason why their calculated ground state is a triplet configuration.

In $C_{4 v}$ symmetry we get a lateral bond length of 2.306 $\AA$, a lateral-axial bond length of $2.491 \AA$ and the configuration ${ }^{1} A_{1}, \quad\left(1 a_{1}\right)^{2}(1 e)^{4}\left(2 a_{1}\right)^{2}\left(1 b_{2}\right)^{2}\left(1 b_{1}\right)^{2}\left(3 a_{1}\right)^{2}$ $(2 e)^{4}\left(4 a_{1}\right)^{2}$, in almost perfect agreement with Raghavachari $^{11}$ whose atomic distances are 2.295 and $2.502 \AA$, respectively. Once more, the results of PK (Ref. 12) have to be discarded because of their assumption of equal bond lengths. The overlap population of $0.695 e$ between two lateral atoms versus $0.329 e$ between the axial atom and one lateral atom indicates much stronger covalent bonding within the square. The axial atom is negatively charged by $-0.08 e$ leading to positive charges of $0.02 e$ at
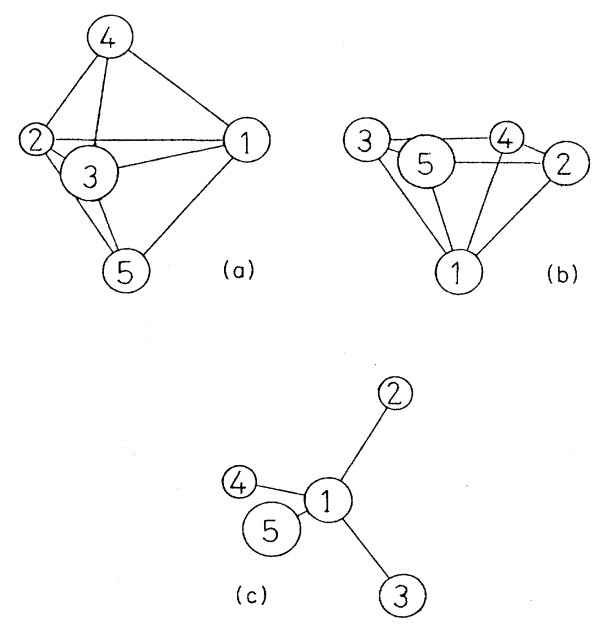

FIG. $1 . \mathrm{Si}_{5}$ cluster in (a) $D_{3 h}$ symmetry (trigonal bipyramid) with the lateral atoms $1,2,3$ and the axial atoms 4,5 ; (b) $C_{4 v}$ symmetry with the axial atom 1 and the lateral atoms $2,3,4,5$; (c) $T_{d}$ symmetry with the central atom 1 and the peripheral atoms $2,3,4,5$. the lateral sites.

In tetrahedral geometry we compute ${ }^{1} A_{1}$, $\left(1 a_{1}\right)^{2}\left(1 t_{2}\right)^{6}\left(2 a_{1}\right)^{2}\left(2 t_{2}\right)^{6}(1 e)^{4}$, as the ground-state valence-electron configuration with a bond length of $2.171 \AA$. The calculation of PK (Ref. 12) yields $2.50 \AA$ in a ${ }^{5} E$ ground-state configuration, both results appearing rather unlikely. In agreement with PK (Ref. 12) we deduce from the overlap population of $0.82 e$ between the central atom and a peripheral atom that these interactions constitute the stability of tetrahedral $\mathrm{Si}_{5}$, the overlap charge between two peripheral atoms being only $0.01 e$. The effective charges of 0.128 e for the central atom and $-0.032 e$ for each peripheral atom due to internal charge fluctuations should slightly stabilize this cluster and shorten the bond length. The smooth potential curve of $\operatorname{Si}_{5}\left(T_{d}\right)$ shows an extremely weak bonding and Raghavachari and Logovinsky ${ }^{14}$ suppose this cluster even to be unstable. This conclusion, however, could be due to the neglect of the Coulomb correlations in the HartreeFock calculations becoming especially important when strong repulsive nonbonding interactions between lonepair orbitals occur, as is also the case in $F_{2}$.

We obtain the result that $\mathrm{Si}_{5}$ is most stable in $D_{3 h}$ symmetry which agrees with Raghavachari ${ }^{11}$ but not with PK (Ref. 12) who favor a planar $C_{2 v}$ geometry, maybe due to unreasonable geometry restrictions in the case of $D_{3 h}$ and $C_{4 v}$. Therefore, $D_{3 h}$ is proposed to be the ground-state geometry for $\mathrm{Si}_{5}$.

Altogether, the SCC- $X \alpha$ bond lengths agree with the Hartree-Fock results of Raghavachari ${ }^{11}$ within $5 \%$ or better which proves that $\mathrm{Si}-\mathrm{Si}$ bonds are well described in the framework of the SCC- $X \alpha$ method and that reliable results can be expected also for calculations on the silicon surface.

\section{THE CLEAN SI(111) SURFACE}

The solid-state model and the cluster model are two approaches to treat adsorption phenomena on solid surfaces, which mainly differ in modeling the substrate. The solid-state model assumes it as a two-dimensional ideal surface only weakly disturbed by the adsorbate. For several reasons, viz., problems with self-consistency, nonideal substrate surfaces and determination of adsorption geometries via total-energy calculations, the solidstate model is not a suitable approach in our case. Within the cluster model the adsorption site and its environment are represented by a finite cluster; this approach 
is applicable if the adsorption can be assumed to be in good approximation a local phenomenon. ${ }^{15}$ Although the cluster model is less realistic, it offers several practical advantages compared to the solid-state model concerning the treatment of nonideal surfaces and the determination of adsorption geometries, in particular. The well-known drawbacks of the cluster model, viz., the size problem, internal charge fluctuations, and the embedding problem can be treated by standard techniques described, e.g., in Hütsch. ${ }^{9}$

The only theoretical work on a $\mathrm{Si}(111)-7 \times 7$ surface reconstructed in the DAS-model is a recent calculation of Qian and Chadi ${ }^{16}$ which is, however, non-self-consistent and restricted to next-neighbor interactions. Even though the simplicity of this approach allows the handling of a cluster of $200 \mathrm{Si}$ atoms and $49 \mathrm{H}$ atoms, and determination of its equilibrium geometry, it precludes, e.g., the distinction between a fcc and a hcp lattice since for tetrahedral coordinated atoms the next-neighbor environments are equal in both cases. Thus, the stacking fault in the DAS model cannot be accounted for but has to be introduced as an assumption.

In the SCC- $X \alpha$ method $^{3}$ the interatomic-potential matrix elements are not parametrized but explicitly calculated through

$$
V_{i j}^{\mu v}=\int d^{3} r \varphi_{i}^{(\mu)}\left(\mathbf{r}-\mathbf{R}_{\mu}\right)\left[\sum_{\mu \neq v} V_{\mathrm{at}}^{(\kappa)}\left(\mathbf{r}-\mathbf{R}_{\kappa}\right)\right] \varphi_{j}^{(v)}\left(\mathbf{r}-\mathbf{R}_{v}\right),
$$

where $\varphi_{i}^{(\mu)}\left(\mathbf{r}-\mathbf{R}_{\mu}\right)$ denotes the $\left(n_{i} l_{i} m_{i}\right)$ real atomic valence orbital of the atom $\mu$ located at $R_{\mu}$. The atomic potential $V_{\text {at }}^{(\kappa)}$ is described in the $X \alpha$ approximation, and the sum includes all cluster atoms $\kappa$, which removes the restriction to next-neighbor interactions. Moreover, the resulting secular equation is solved self-consistently with respect to atomic-orbital $(\mathrm{AO})$ occupation numbers

$$
x_{i}^{\mu}=2 a_{i}^{\mu} \sum_{v, j} S_{i j}^{\mu v} P_{i j}^{\mu v} /\left(a_{i}^{\mu}+a_{j}^{v}\right),
$$

which are constructed from the overlap matrix $S_{i j}^{\mu v}$ and the bond-order matrix elements

$$
P_{i j}^{\mu v}=\sum_{k} n_{k} c_{i k}^{\mu} c_{j k}^{v}
$$

where $n_{k}$ are the occupation numbers of the molecular orbitals (MO's) and the weight factors ${ }^{17}$ are introduced in order to overcome the drawbacks of the Mulliken population analysis in a simple way. Whereas the SCC-X $\alpha$ calculation should yield more reliable results, it imposes limitations upon the cluster size for handling the system within reasonable computation time. In our case, the minimal cluster size is determined by the requirement of including at least the next nearest neighbors of each adsorption site because it has turned out that these are still affected by $\mathrm{Ge}$ adsorption. Consequently, we selected a $\mathrm{Si}_{28} \mathrm{H}_{26}$ cluster (cf. Fig. 2) to model the $\mathrm{Si}(111)-7 \times 7$ surface as the substrate for $\mathrm{Ge}$ adsorption. As usual, the $\mathrm{H}$ atoms serve to saturate the $\mathrm{Si}$ bonds on the artificial surface arising from cutting the cluster out of the bulk. The average effective charge of all $\mathrm{Si}$ bulk atoms including

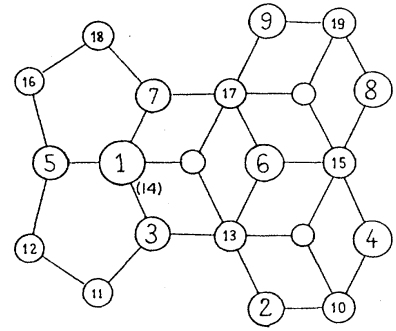

(a)

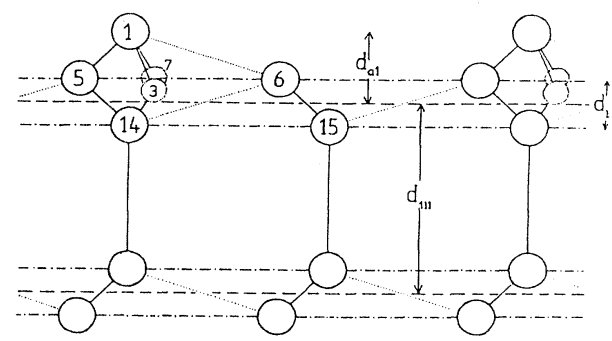

(b)

FIG. 2. (a) Top view of the $\mathrm{Si}$ atoms of the free $\mathrm{Si}_{28} \mathrm{H}_{26}$ cluster with the adatom 1 and the surface site 6 . The second-layer atom 14 under the adatom is hidden from view. (b) Side view of the adatom layer and the first four layers of the unrelaxed $\mathrm{Si}(111)$ surface within a plane determined by atoms $1,5,6$, and 14. The diffraction plane $d_{111}$ is the middle of the Si bilayer and $d_{\mathrm{bi}}$ is the bilayer separation. The computed distance $d_{a 2}$ between the second layer and the adatom layer is $0.67 \AA$ shorter than $d_{111}$. There is no direct bonding between the adatom 1 and the surface-site atom 6 . The atoms 3 and 7 directly bonded to the adatom are above and below the plane (indicated by dotted circles).

those of the artificial surface amounts to $(0.031 \pm 0.031) e$ so that the internal charge fluctuations are sufficiently suppressed. The computation time for this 138-valenceorbital cluster amounts to $95 \mathrm{sec}$ per iteration on a Siemens 7.882 computer, which is approximately $8-10$ times slower than a Cray 1.

Starting from the equilibrium geometry as calculated by Qian and Chadi ${ }^{16}$ we have checked the equilibrium positions of those $\mathrm{Si}$ atoms where $\mathrm{Ge}$ adsorption takes place, namely the surface site [atom 6, cf. Fig. 2(a)] and the adatom (atom 1). Defining the surface as the average $z$ coordinate of those surface atoms [atoms $2,3,4,5,7,8$, and 9, cf. Fig. 2(a)] saturated by an adatom in the DAS model, the height of the adatom is $1.266 \AA$ corresponding to an average bond distance of $2.461 \AA$ to the neighboring atoms 3,5 , and 7 . This is in almost perfect agreement with the results of Qian and Chadi, ${ }^{16}$ our bond lengths being only $0.028 \AA$ shorter. On the other hand, the height of the surface site over the surface is $0.068 \AA$, giving an average bond length to its next-neighbor atoms 13 , 15 , and 17 of $2.310 \AA$, which is shorter by $0.100 \AA$ than the respective value of Qian and Chadi. ${ }^{16}$ This does not seem to be a large deviation but the corresponding heights of atom 6 over the surface differ significantly, Chadi and Qian's ${ }^{16}$ surface site protruding by $0.261 \AA$ over the surface. However, it should be noticed that our 
calculated distance is slightly closer to the Si bulk bond length of $2.35 \AA$.

The reason for the differences for these two sites could originate from higher-order neighbor interactions. For the surface atom is in a real "hollow site" with an atom underneath only in the sixth layer, whereas the adatom resides essentially in a "top site" because the atom 14 [hidden from view in Fig. 2(a) by the adatom] is located directly below at a distance of $2.44 .1 \AA$. For decreasing distances the adatom will be prevented from sinking into the bulk by atom 14 while such an argument does not apply to the surface site. This effect, however, can only be taken into account if there is no restriction to interaction between $s p^{3}$-bonded partners. Northrup ${ }^{18}$ and Qian and Chadi ${ }^{16}$ calculate the distance between the adatom and atom 14 to be $2.49 \AA$ (which is $2 \%$ above our value of $2.44 \AA$ ) while Daum, Ibach, and Müller ${ }^{19}$ obtain $2.32 \AA$ (which is $5 \%$ below). From the short separation one cannot, however, conclude that the adatom is fivefold coordinated, as do Daum, Ibach, and Müller. ${ }^{19}$ We obtain a bond with predominant $\left(p_{z} p_{z} \sigma\right)$ character and an overlap population of $0.124 e$, significantly lower than the usual Si-Si overlap population in the bulk of approximately $0.6 e$. Such a bonding is also consistent with the results of Northrup ${ }^{18}$ who computed the total energy per adatom in a top site as being $0.64 \mathrm{eV}$ lower than in a hollow site.

Our results can be eventually made compatible with a recent $\mathrm{x}$-ray standing-wave (XSW) measurement by Durbin et al. ${ }^{20}$ They interpreted the experiment in another way, namely as a contraction in the [111] direction of the topmost two atom layers by $0.57 \pm 0.16 \AA$. This did not include the adatom layer as the topmost one, since they assume the adatoms to be of no influence within their error bars. If the experiment is granted as being correct it can be reinterpreted along the following line of argument. We think that Durbin et al. ${ }^{20}$ could have erroneously believed that they had measured the distance between the surface and the third layer instead of the distance between the adatom layer and the second layer. They attribute an Auger-electron yield proportional to $e^{-D_{i} / \lambda}$ to the $i$ th atom layer of the depth $D_{i}$ where the mean free path $\lambda$ of an Auger electron is assumed to be layer independent. Since the adatom layer consists of only 12 adatoms per $7 \times 7$ surface unit cell, a mean free path for that layer about four times larger should be a better approximation, resulting in an accordingly larger Augerelectron yield, not too different from that of a bulk layer. Our computed adatom height over the second layer is $0.67 \AA$ shorter than the $d_{111}$ lattice constant of $3.14 \AA$ appearing, thus, as the contraction of two planes by $0.57 \pm 0.16 \AA$ as measured by Durbin et al. ${ }^{20}$ [cf. Fig. 2(b)].

Table II shows the highest MO's of the electronic structure of our $\mathrm{Si}_{28} \mathrm{H}_{26}$ cluster. The surface atoms 2, 4, 8 , and 9 (cf. Fig. 2) give rise to four delocalized artificial surface states because they are not bonded to an adatom as in the real-surface DAS model. These delocalized

TABLE II. Electronic structure of $\mathrm{Si}_{28} \mathrm{H}_{26}$ (cf. Fig. 2).

\begin{tabular}{|c|c|c|c|c|c|c|c|c|}
\hline $\begin{array}{c}\mathrm{MO} \\
V_{I}(\mathrm{eV})\end{array}$ & $\begin{array}{c}65 \\
7.405\end{array}$ & $\begin{array}{c}\mathrm{AO} \\
66 \\
7.333\end{array}$ & $\begin{array}{c}\text { ibution } \\
67 \\
6.507\end{array}$ & $\begin{array}{c}\text { MO } \\
68 \\
6.465\end{array}$ & $\begin{array}{c}\text { \% of } \mathrm{N} \\
69 \\
6.349\end{array}$ & $\begin{array}{c}70 \\
6.324\end{array}$ & $\begin{array}{c}71 \\
6.285\end{array}$ & $\begin{array}{c}72 \\
5.465\end{array}$ \\
\hline $\begin{array}{l}1 \mathrm{Si} 3 s^{\mathrm{a}} \\
1 \mathrm{Si} 3 p^{\mathrm{a}}\end{array}$ & $\begin{array}{l}0 \\
7\end{array}$ & $\begin{array}{r}0 \\
10\end{array}$ & & & & & & $\begin{array}{r}8 \\
31\end{array}$ \\
\hline $\begin{array}{l}2+9 \mathrm{Si} 3 s^{\mathrm{b}} \\
2+9 \mathrm{Si} 3 p^{\mathrm{b}}\end{array}$ & & & $\begin{array}{r}8 \\
42\end{array}$ & $\begin{array}{r}7 \\
42\end{array}$ & $\begin{array}{r}5 \\
25\end{array}$ & $\begin{array}{r}6 \\
29\end{array}$ & $\begin{array}{l}0 \\
3\end{array}$ & \\
\hline $\begin{array}{l}4+8 \text { Si } 3 s^{b} \\
4+8 \text { Si } 3 p^{b}\end{array}$ & & & $\begin{array}{r}4 \\
21\end{array}$ & $\begin{array}{r}5 \\
27\end{array}$ & $\begin{array}{r}9 \\
46\end{array}$ & $\begin{array}{r}9 \\
47\end{array}$ & $\begin{array}{l}0 \\
3\end{array}$ & \\
\hline $\begin{array}{l}3+7 \mathrm{Si} 3 s^{\mathrm{c}} \\
3+7 \mathrm{Si} 3 p^{\mathrm{c}}\end{array}$ & $\begin{array}{r}2 \\
51\end{array}$ & $\begin{array}{r}0 \\
17\end{array}$ & & & & & & $\begin{array}{r}3 \\
26\end{array}$ \\
\hline $\begin{array}{lll}5 & \mathrm{Si} & 3 s^{\mathrm{d}} \\
5 & \mathrm{Si} & 3 p^{\mathrm{d}}\end{array}$ & $\begin{array}{l}0 \\
1\end{array}$ & $\begin{array}{r}2 \\
35\end{array}$ & & & & & & $\begin{array}{r}2 \\
12\end{array}$ \\
\hline $\begin{array}{l}6 \mathrm{Si} 3 s^{\mathrm{e}} \\
6 \mathrm{Si} 3 p^{\mathrm{e}}\end{array}$ & & & $\begin{array}{l}2 \\
8\end{array}$ & & & & $\begin{array}{r}8 \\
63\end{array}$ & \\
\hline $\begin{array}{l}14 \mathrm{Si} 3 s^{\mathrm{f}} \\
14 \mathrm{Si} 3 p^{\mathrm{f}} \\
\end{array}$ & $\begin{array}{r}0 \\
28 \\
\end{array}$ & $\begin{array}{r}0 \\
28 \\
\end{array}$ & & & & & & $\begin{array}{l}1 \\
1\end{array}$ \\
\hline
\end{tabular}

${ }^{\text {a Adatom. }}$

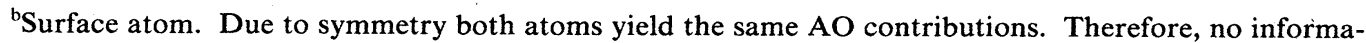
tion is lost by their addition.

${ }^{\mathrm{c}}$ Surface atom saturated by adatom. Due to symmetry both atoms yield the same AO contributions. Therefore, no information is lost by their addition.

${ }^{\mathrm{d}}$ Surface atom saturated by adatom.

${ }^{\text {eSurface site. }}$

${ }^{\text {f }}$ Second-layer atom under adatom. 
artificial surface states (MO's 67-70) are easily identified by their atom-orbital (AO) composition in Table II, which displays all contributions $\geq 1 \%$ to the MO's. MO 72 is the adatom surface state, first observed by Binnig et $a l .{ }^{21}$ with scanning tunneling microscopy (STM). MO 71 has a $71 \%$ AO contribution from the surface site (atom 6) and corresponds to one of the six (real) surface states of the $7 \times 7$ unit cell first identified by Hamers et $a l .^{22}$ by using the same technique. According to our calculation, the adatom surface state consists of $39 \%$ adatom contributions and 45\% AO contributions from the four next neighbors (atoms 3, 5, 7, and 14; cf. Fig. 2) of the adatom and is, hence, delocalized over a $\mathrm{Si}_{5}$ cluster with a geometry close to that of a trigonal bipyramid calculated in Sec. II.

A further means for testing the suitability of the $\mathrm{Si}_{28} \mathrm{H}_{26}$ cluster for modeling the $\mathrm{Si}(111)-7 \times 7$ surface is supplied by the comparison of our calculated ionization potentials $V_{I}$ with other experimental ${ }^{22}$ and theoretical ${ }^{16}$ values. The ionization potentials are given relative to the Fermi energy $E_{F}$, defined as the energy of the highest occupied state. The surface states at the adatom (MO 72) and at the surface site (MO 71) as well as the backbond (MO's 65 and 66) are most suitable for this comparison. Since the backbond MO's are nearly degenerate we define their $V_{I}$ as

$$
\begin{aligned}
V_{I}(\text { backbond }) \equiv & \frac{V_{I}(\mathrm{MO} 66)+V_{I}(\mathrm{MO} \mathrm{65})}{2} \\
& \pm \frac{V_{I}\left(\mathrm{MO} \mathrm{66)}-V_{I}(\mathrm{MO} \mathrm{65})\right.}{2} .
\end{aligned}
$$
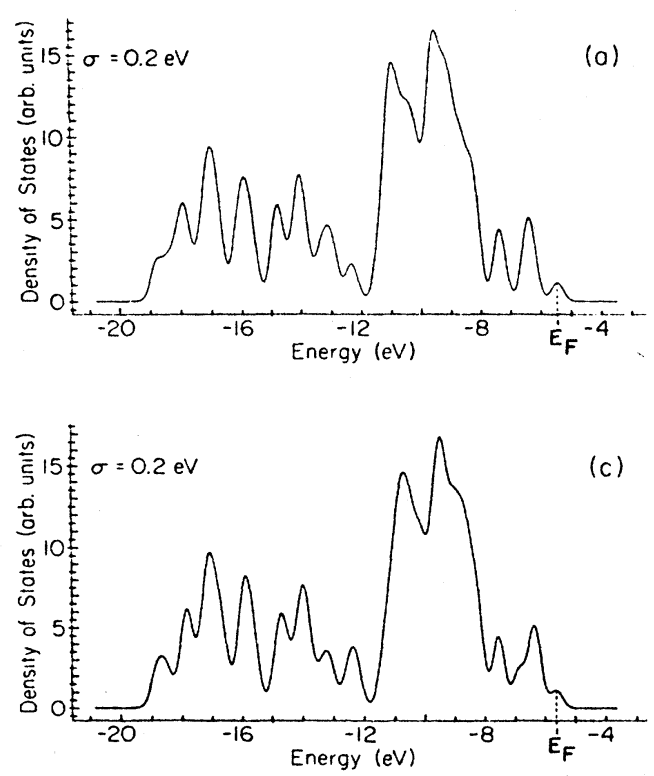

The values for the MO's obtained in this way are given in Table III. The $V_{I}$ calculated with the SCC- $X \alpha$ method match the experimental data slightly better than those of Qian and Chadi. ${ }^{16}$ However, with respect to the size of the investigated systems, the maximum deviation of 0.2 $\mathrm{eV}$ has to be regarded as a good mutual agreement between both modes of calculation.

Considering the density of states (DOS) permits the qualitative comparison with photoelectron spectra (PES) when the discrete levels $\varepsilon_{k}$ are folded by a Gaussian according to ${ }^{23}$

$$
N(\varepsilon)=\sum_{k} \frac{n_{k}}{\sqrt{2 \pi} \sigma} e^{-(1 / 2)\left[\left(\varepsilon-\varepsilon_{k}\right) / \sigma\right]^{2}}
$$

The DOS for the cluster $\mathrm{Si}_{28} \mathrm{H}_{26}$ is shown in Fig. 3(a). The peak at $E_{F}$ is attributed to the adatom surface state and the peak at about $-6.3 \mathrm{eV}$ arises from the surface site. The next peak at $-7.5 \mathrm{eV}$ comes from dangling bonds of the surface atoms 2, 4, 8, and 9 and is, therefore, a cluster artifact. The real surface states agree with the ultraviolet PES results from Demuth et al. ${ }^{24}$ More than qualitative agreement in the bulk region cannot be expected because, first, a cluster of only $28 \mathrm{Si}$ atoms is not sufficient and second, Eq. (4) does not incorporate cross sections.

To assess the influence of the artificial dangling bonds of the surface atoms $2,4,8$, and 9 on the results so far obtained, the position of the surface site atom was varied again in a $\mathrm{Si}_{28} \mathrm{H}_{30}$ cluster where these bonds were each saturated by one $\mathbf{H}$ atom. In this case, the surface site atom moves closer to the surface by $0.002 \AA$ (a negligible
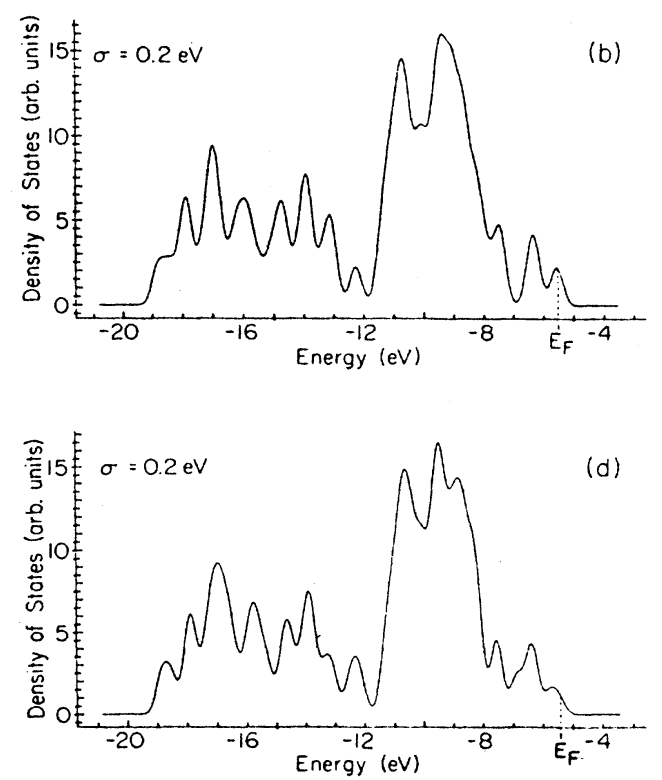

FIG. 3. (a) Density-of-states curve for the free $\mathrm{Si}_{28} \mathrm{H}_{26}$ cluster. Here and in the subsequent DOS plots, $E_{F}$ is defined as the energy of the highest occupied state. (b) DOS curve for the $\mathrm{Si}_{28} \mathrm{H}_{26}$ cluster with one Ge atom adsorbed on the surface site (atom 6 in Fig. 2). (c) DOS curve for the $\mathrm{Si}_{28} \mathrm{H}_{26}$ cluster with one $\mathrm{Ge}$ atom adsorbed on the adatom site (atom 1 in Fig. 2). (d) DOS curve of the $\mathrm{Ge}_{2} \mathrm{Si}_{28} \mathrm{H}_{26}$ cluster for coadsorption on the adatom and on the surface-site atom. 
TABLE III. Comparison of selected surface states from various sources.

\begin{tabular}{lclc}
\hline \hline Source & $\begin{array}{c}\text { Hamers } \text { et al. } \\
\text { (Ref. 23) } \\
\text { (expt.) }\end{array}$ & $\begin{array}{c}\text { Qian and Chadi } \\
\text { (Ref. 17) } \\
\text { (theor.) }\end{array}$ & $\begin{array}{c}\text { Present work } \\
\text { State }\end{array}$ \\
\hline Adatom & $E_{F}-0.2 \mathrm{eV}$ & $E_{F}$ & $E_{F}$ \\
Surface site & $E_{F}-0.8 \mathrm{eV}$ & $E_{F}-0.9 \mathrm{eV}$ & $E_{F}-0.82 \mathrm{eV}$ \\
Backbond & $E_{F}-1.7 \mathrm{eV}$ & $E_{F}-1.5 \mathrm{eV}$ & $E_{F}-(1.90 \pm 0.04) \mathrm{eV}$ \\
\hline \hline
\end{tabular}

effect), and the $V_{I}$ of the adatom and the backbond are $E_{F}-0.79 \mathrm{eV}$ and $E_{F}-(1.89 \pm 0.04) \mathrm{eV}$, respectively. In spite of these minor improvements with regard to the experimental data from Hamers et al., ${ }^{22}$ the subsequent calculations on $\mathrm{Ge}$ adsorption on this substrate are performed with the unsaturated $\mathrm{Si}_{28} \mathrm{H}_{26}$ cluster because the changes seem to be too small to justify the additional computational effort for the larger cluster.

\section{Ge ADSORPTION ON A Si(111)- $7 \times 7$ SURFACE}

The last set of calculations comprises the adsorption of a single Ge atom on the surface-atop site [atom 6 in Fig. 2(a)], on the adatom-atop site [atom 1 in Fig. 2(a)], and the coadsorption of two Ge atoms on both sites in the DAS model. These investigations are intended to contribute to the understanding of the initial growth steps of thin Ge layers on silicon.

Dev et al. ${ }^{25,26}$ have grown Ge on a $\mathrm{Si}(111)-7 \times 7$ substrate. According to their XSW measurements the Ge atoms are exclusively adsorbed on surface-atop sites for low coverages [up to $\Theta=0.2$ monolayers (ML); 1 $\mathrm{ML} \cong 49 \mathrm{Ge}$ atoms per $7 \times 7$ unit cell] whereas for $\Theta \approx 0.4$ $\mathrm{ML}, \mathrm{Ge}$ is adsorbed on the $\mathrm{Si}$ adatoms as well. The possibility of $\mathrm{Ge}$ adsorption on $\mathrm{Si}$ adatoms has been questionable so far.

Starting with the adsorption of a single $\mathrm{Ge}$ atom on the surface-atop site of the $\mathrm{Si}_{28} \mathrm{H}_{26}$ cluster, we are comparing the electronic structure, Table IV, with that of the free cluster. The artificial surface states (MO's 69-72 in Table IV) are destabilized by only about $0.1 \mathrm{eV}$ compared to the free cluster (cf. Table II) due to AO contributions of up to $2 \%$ from Ge $p$ orbitals. The nearly degenerate highest occupied MO's 74 and 75 are lone pairs of the adsorbed Ge (atom 55). The dangling bond of the surfacesite $\mathrm{Si}$ atom is pushed into the bulk states due to $\mathrm{Ge}$ adsorption, as can be verified by comparing the DOS curve [Fig. 3(b)] with that of the free cluster [Fig. 3(a)]. The minima in the DOS of the free cluster at -15.3 and $-16.6 \mathrm{eV}$ are less pronounced when $\mathrm{Ge}$ is adsorbed.

The SCC- $X \alpha$ valence-electron total energy as a function of the Si-Ge distance has been determined next. The calculated bond length of $2.638 \AA$ exceeds the Ge bulk bond length of $2.45 \AA$ considerably, most likely for the following reason. There is a spurious $16 \% \mathrm{AO}$ contribution of the Ge atom to MO 73 (cf. Table IV) consisting otherwise of AO's from the trigonal bipyramid structure at the adatom (atoms 1, 3, 5, 7, and 14). We suspect this to be caused by numerical instabilities during matrix diagonalization which may occasionally occur when many eigenvalues are closely spaced. It is quite improbable that there exists a physical reason for this Ge AO contri- bution because of the separation of $4.60 \AA$ between the adatom and the Ge adsorbate, which is also confirmed by the negligible overlap population of $0.008 e$. The effect of this $16 \%$ spurious contribution is an increased amount of electron density attributed to the $\mathrm{Ge}$ atom, yielding an effective negative charge of $-0.02 e$, whereas a slightly positive charge on $\mathrm{Ge}$ should be expected in a $\mathrm{Si}-\mathrm{Ge}$ bond. This argument gets support from another set of calculations where the Ge adsorption on the surface-atop site is repeated with the "saturated" $\mathrm{Si}_{28} \mathrm{H}_{30}$ cluster. This allows us to check the influence of the artificial surface states, too. In fact, this spurious AO contribution vanishes when the Si substrate is modified in this way. However, these calculations could not be made convergent for all geometries since three almost-degenerate MO's interchange during the iteration for some Si-Ge distances.

The next series of calculations investigates whether a Ge atom (atom 55) can be adsorbed on the adatom-atop site (atom 1). A minimum in the SCC- $X \alpha$ total valence energy as a function of the Si-Ge distance indicates clearly a stable bond. The electronic structure of the substrate-adsorbate system is given in Table V, and Fig. 3(c) shows the density-of-states curve. As expected, the free-cluster surface state at the adatom (MO 72 in Table II) is pushed into the bulk states upon Ge adsorption on the adatom. The calculated equilibrium distance between the adatom and the adsorbed $\mathrm{Ge}$ is $2.339 \AA$ and thus $0.299 \AA$ shorter than the corresponding bond length for surface-atop site adsorption. The overlap population of $0.842 e$ between the adsorbed $\mathrm{Ge}$ and the $\mathrm{Si}$ adatom is distinctly larger than for surface-atop adsorption with an overlap population of $0.641 e$. However, this difference can be attributed mainly to the different bond lengths and does not tell anything about the preferred adsorption site. To find the energetically favorable adsorption site, one has to compare the total energies. For a single adsorbed Ge atom we obtain a value for the total energy $2.502 \mathrm{eV}$ per atom or $57.69 \mathrm{kcal} / \mathrm{mol}$ lower for surface-atop adsorption than for adatom-atop adsorption. This value seems reasonable with respect to the bulk cohesive energies for $\mathrm{Si}(176 \mathrm{kcal} / \mathrm{mol})$ and $\mathrm{Ge}(159 \mathrm{kcal} / \mathrm{mol})$ even though we assume the true energy difference to be smaller by a factor of roughly 1.5 because $X \alpha$ procedures tend to overestimate binding energies in open-shell systems ${ }^{27}$ like the substrate-adsorbate system dealt with here. The difference in total energy can easily be understood by comparing the two DOS curves for single adsorption. In the case of surface-atop adsorption [cf. Fig. 3(b)], a smaller average density of states is recognized in the region from $E_{F}$ down to roughly $E_{F}-13 \mathrm{eV}$ than for adatomatop adsorption [cf. Fig. 3(c)], whereas the opposite is found in the interval between $E_{F}-13 \mathrm{eV}$ and $E_{F}-19 \mathrm{eV}$. 
TABLE IV. Electronic structure of $\mathrm{Si}_{28} \mathrm{H}_{26}$ for adsorption of a single $\mathrm{Ge}$ atom (atom 55) on the surface site (atom 6).

\begin{tabular}{|c|c|c|c|c|c|c|c|}
\hline & & $O$ cont & ons to & (in $\%$ & & & \\
\hline MO & 69 & 70 & 71 & 72 & 73 & 74 & 75 \\
\hline$V_{I}(\mathrm{eV})$ & 6.431 & 6.429 & 6.294 & 6.264 & 5.601 & 5.545 & 5.534 \\
\hline $1 \mathrm{Si} 3 s^{\mathrm{a}}$ & & & & & 6 & & 2 \\
\hline $1 \mathrm{Si} 3 p^{\mathrm{a}}$ & & & & & 26 & & 7 \\
\hline $2+9 \mathrm{Si} 3 s^{\mathrm{b}}$ & 9 & 8 & 4 & 4 & & 0 & \\
\hline $2+9 \mathrm{Si} 3 p^{\mathrm{b}}$ & 49 & 49 & 21 & 23 & & 2 & \\
\hline $4+8 \mathrm{Si} 3 s^{\mathrm{b}}$ & 3 & 3 & 9 & 10 & & 0 & 0 \\
\hline $4+8 \mathrm{Si} 3 p^{\mathrm{b}}$ & 18 & 20 & 50 & 53 & & 1 & 1 \\
\hline $3+7 \mathrm{Si} 3 s^{\mathrm{c}}$ & & & & & 3 & & 0 \\
\hline $3+7 \mathrm{Si} 3 p^{\mathrm{c}}$ & & & & & 24 & & 4 \\
\hline $5 \mathrm{Si} 3 s^{\mathrm{d}}$ & & & & & 1 & & 1 \\
\hline $5 \mathrm{Si} 3 p^{\mathrm{d}}$ & & & & & 9 & & 4 \\
\hline $14 \mathrm{Si} 3 s^{\mathrm{e}}$ & & & & & 1 & & \\
\hline $14 \mathrm{Si} 3 p^{\mathrm{e}}$ & & & & & 1 & & \\
\hline $55 \mathrm{Ge} 4 s^{\mathrm{f}}$ & 0 & 0 & 0 & & 0 & 0 & 0 \\
\hline $55 \mathrm{Ge} 4 p^{\mathrm{f}}$ & 1 & 2 & 1 & & 16 & 95 & 74 \\
\hline
\end{tabular}

${ }^{\text {a Adatom. }}$

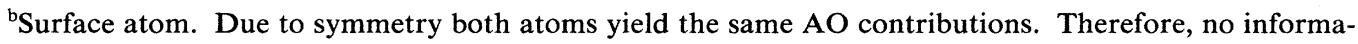
tion is lost by their addition.

${ }^{\mathrm{c}}$ Surface atom saturated by adatom. Due to symmetry both atoms yield the same AO contributions. Therefore, no information is lost by their addition.

${ }^{\mathrm{d}}$ Surface atom saturated by adatom.

'Second-layer atom under adatom.

${ }^{f}$ Germanium adsorbate.

TABLE V. Electronic structure of $\mathrm{Si}_{28} \mathrm{H}_{26}$ for adsorption of a single Ge atom (atom 55) on the adatom (atom 1).

\begin{tabular}{|c|c|c|c|c|c|c|c|}
\hline \multicolumn{8}{|c|}{ AO contributions to $\mathrm{MO}$ (in \% of $\mathrm{MO}$ ) } \\
\hline MO & 69 & 70 & 71 & 72 & 73 & 74 & 75 \\
\hline$V_{I}(\mathrm{eV})$ & 6.478 & 6.435 & 6.320 & 6.296 & 6.250 & 5.635 & 5.633 \\
\hline $2+9 \mathrm{Si} 3 s^{\mathrm{a}}$ & 8 & 7 & 5 & 6 & 0 & & \\
\hline $2+9 \mathrm{Si} 3 p^{\mathrm{a}}$ & 43 & 44 & 25 & 29 & 4 & & \\
\hline $4+8 \mathrm{Si} 3 s^{\mathrm{a}}$ & 4 & 5 & 9 & 9 & 0 & & \\
\hline $4+8 \mathrm{Si} 3 p^{\mathrm{a}}$ & 21 & 27 & 47 & 47 & 2 & & \\
\hline $3+7 \mathrm{Si} 3 s^{\mathrm{b}}$ & & & & & & 2 & 0 \\
\hline $3+7 \mathrm{Si} 3 p^{\mathrm{b}}$ & & & & & & 8 & 3 \\
\hline $5 \mathrm{Si} 3 s^{\mathrm{c}}$ & & & & & & & 1 \\
\hline $5 \mathrm{Si} 3 p^{\mathrm{c}}$ & & & & & & & 5 \\
\hline $6 \mathrm{Si} 3 s^{\mathrm{d}}$ & 1 & & & & 8 & & \\
\hline $6 \mathrm{Si} 3 p^{\mathrm{d}}$ & 8 & & & & 63 & & \\
\hline $55 \mathrm{Ge} 4 s^{\mathrm{e}}$ & & & & & & 0 & 0 \\
\hline $55 \mathrm{Ge} 4 p^{\mathrm{e}}$ & & & & & & 89 & 88 \\
\hline
\end{tabular}

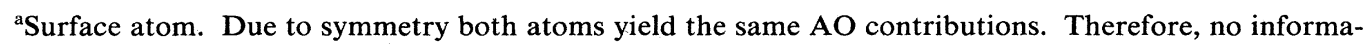
tion is lost by their addition.

burface atom saturated by adatom. Due to symmetry both atoms yield the same AO contributions. Therefore, no information is lost by their addition.

'Surface atom saturated by adatom.

${ }^{\mathrm{d}}$ Surface site.

${ }^{\text {e }}$ Germanium adsorbate. 
Thus, surface-atop adsorption of a $\mathrm{Ge}$ atom diminishes the density of surfacelike states in favor of a higher density of bulklike states to a larger extent than adatom-atop adsorption, leading eventually to a lower valence total energy in the former case.

Next, we turn to the coadsorption of Ge on the surface site and the adatom site. The electronic structure is given in Table VI, and Fig. 3(d) shows the DOS curve. The $V_{I}$ of the artificial dangling bonds (MO's 71-74 in Table VI) are nearly unchanged if compared with both cases of single adsorption as well as to the bare cluster so that noticeable effects on the computed equilibrium geometry are not expected. The next four MO's 75-78 are Ge lone pairs with the lower two mainly located at the surfaceatop-site-adsorbed $\mathrm{Ge}$ (atom 55) whereas the upper ones belong to the adatom-atop Ge (atom 56). With $85 \%$ AO contribution from atom 55, MO 76 is identified as the surface-atop adsorbate level and, analogously, MO 77 with $82 \%$ Ge $p$-orbital contributions is a lone pair from the $\mathrm{Ge}$ atom adsorbed on the adatom. The situation is less clear with MO's 75 and 78 because MO 75 contains a $34 \%$ contribution from atom 56, although the corresponding Ge lone pair MO 77 has a $0.264 \mathrm{eV}$ higher $V_{I}$. Likewise, MO 78 exhibits a $40 \%$ p-orbital contribution of atom 55 adsorbed on the surface site with its "true" lone pair MO 76 lower by $0.290 \mathrm{eV}$. This is striking because for adsorption of a single $\mathrm{Ge}$ atom the lone pairs are nearly degenerate, i.e., for surface-atop adsorption the separation between the Ge lone pairs is $0.011 \mathrm{eV}$ (MO's 74 and 75 in Table IV) and for adatom-atop adsorption the splitting is only $0.002 \mathrm{eV}$ (MO's 74 and 75 in Table V).
The various bonds between the substrate and the adsorbate can be analyzed by comparing the dominant contributions that constitute the bond (cf. Table VII) with each other. Two points should be emphasized. First, the $\mathrm{Ge}-\mathrm{Si}$-adatom bond has a significantly larger $\pi$ character and, second, the bond populations for single adsorption and coadsorption, respectively, are almost identical in both cases.

The effective charge of the surface-atop $\mathrm{Ge}$ atom is more positive by $0.029 e$ than that of the adatom-atop one which explains the ordering of the Ge lone-pair MO's. This charge difference can be understood in terms of the larger distance of the $\mathrm{Ge}$ atom on the $\mathrm{Si}$ adatom site to the average surface (defined by the atoms $2,3,4,5,7,8$, and 9; cf. Sec. III) since a larger distance leads to a decreased charge separation between the surface and the adsorbed atom that reflects the experimental situation, too. The computed effective $\mathrm{Ge}$ charges and $\mathrm{Si}-\mathrm{Ge}$ bond lengths are as follows.

\begin{tabular}{lccc} 
& \multicolumn{2}{c}{ Coadsorption } & Single ads. \\
\hline Adsorption on & $Q_{\text {eff }}(\mathrm{Ge})$ & $d(\mathrm{Si}, \mathrm{Ge})$ & $d(\mathrm{Si}, \mathrm{Ge})$ \\
Adatom-atop & $0.015 e$ & $2.312 \AA$ & $2.339 \AA$ \\
Surface-atop & $0.044 e$ & $2.515 \AA$ & $2.638 \AA$
\end{tabular}

Whereas the $\mathrm{Si}$-adatom-Ge bond length remains almost unchanged for coadsorption, a considerable shortening by $0.123 \AA$ of the $\mathrm{Si}-\mathrm{Ge}$ bond length is obtained in the case of surface-atop adsorption. This confirms our earlier assumption that the unreasonably large distance of $2.638 \AA$ may be due to a numerical instability.

By investigating, finally, the additional adsorption of a

TABLE VI. Electronic structure of $\mathrm{Si}_{28} \mathrm{H}_{26}$ for coadsorption of two Ge atoms. Atom $55 \mathrm{Ge}$ is adsorbed on the surface site (atom 6) and atom $56 \mathrm{Ge}$ is adsorbed on the adatom (atom 1).

\begin{tabular}{|c|c|c|c|c|c|c|c|c|}
\hline & & $\mathrm{AO}$ & ibution & MO & $\%$ of $\mathrm{M}$ & & & \\
\hline MO & 71 & 72 & 73 & 74 & 75 & 76 & 77 & 78 \\
\hline$V_{I}(\mathrm{eV})$ & 6.463 & 6.459 & 6.335 & 6.305 & 5.889 & 5.713 & 5.625 & 5.423 \\
\hline $2+9 \mathrm{Si} 3 s^{\mathrm{a}}$ & 6 & 6 & 7 & 5 & & 0 & & \\
\hline $2+9$ Si $3 p^{\mathrm{a}}$ & 39 & 37 & 33 & 31 & & 1 & & \\
\hline $4+8 \mathrm{Si} 3 s^{\mathrm{a}}$ & 5 & 5 & 7 & 8 & 0 & 0 & & \\
\hline $4+8 \mathrm{Si} 3 p^{\mathrm{a}}$ & 29 & 29 & 39 & 45 & 2 & 1 & & \\
\hline $3+7$ Si $3 s^{b}$ & & & & & 0 & 0 & 2 & 0 \\
\hline $3+7$ Si $3 p^{\mathrm{b}}$ & & & & & 3 & 1 & 7 & 1 \\
\hline $5 \mathrm{Si} 3 s^{\mathrm{c}}$ & & & & & 1 & & & 0 \\
\hline $5 \mathrm{Si} 3 p^{\mathrm{c}}$ & & & & & 3 & & & 3 \\
\hline $55 \mathrm{Ge} 4 s^{\mathrm{d}}$ & 0 & 0 & 0 & & 0 & 0 & 0 & 0 \\
\hline $55 \mathrm{Ge} 4 p^{\mathrm{d}}$ & 4 & 2 & 1 & & 53 & 85 & 7 & 40 \\
\hline $56 \mathrm{Ge} 4 s^{\mathrm{e}}$ & & & & & 0 & 0 & 0 & 0 \\
\hline $56 \mathrm{Ge} 4 p^{\mathrm{e}}$ & & & & & 34 & 6 & 82 & 53 \\
\hline
\end{tabular}

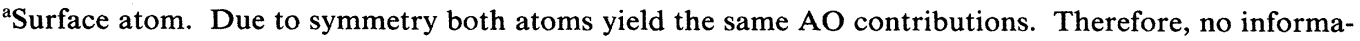
tion is lost by their addition.

burface atom saturated by adatom. Due to symmetry both atoms yield the same AO contributions. Therefore, no information is lost by their addition.

${ }^{\mathrm{c}}$ Surface atom saturated by adatom.

${ }^{\mathrm{d}}$ Germanium adsorbate on the surface site.

${ }^{\mathrm{e}}$ Germanium adsorbate on the adatom. 
TABLE VII. Dominant contributions to the overlap population of the various $\mathrm{Si}-\mathrm{Ge}$ adsorption bonds.

\begin{tabular}{lcccc}
\hline \hline & \multicolumn{2}{c}{ Adatom adsorption } & \multicolumn{2}{c}{ Surface-site adsorption } \\
Bond & Single & Coadsorption & Single & Coadsorption \\
\hline Si $3 p, \mathrm{Ge} 4 p, \sigma)$ & 0.37 & 0.37 & 0.47 & 0.47 \\
$(\mathrm{Si} 3 s, \mathrm{Ge} 4 p, \sigma)$ & 0.25 & 0.25 & 0.16 & 0.18 \\
$(\mathrm{Si} 3 p, \mathrm{Ge} 4 s, \sigma)$ & 0.12 & 0.12 & 0.03 & 0.06 \\
$(\mathrm{Si} 3 p, \mathrm{Ge} 4 p, \pi)$ & 0.13 & 0.14 & 0.01 & 0.02 \\
\hline \hline
\end{tabular}

third Ge atom, we could show that breaking up the adatom structure yields a stable structure according to the experimentally observed transformation of the $\mathrm{Si}(111)$ $7 \times 7$ superstructure into a perfect $1 \times 1$ surface upon $\mathrm{Ge}$ adsorption. ${ }^{28,29}$ Thereby, two Ge atoms are adsorbed on the Si atoms 3 and 5 (cf. Fig. 2) in the same way as the remaining $\mathrm{Ge}$ atom is in the surface-atop site. The former Si adatom binds in an analogous manner to atom 7, albeit at a shorter distance. The calculated bond lengths are $2.386 \AA$ for the $\mathrm{Si}-\mathrm{Ge}$ bond and $1.912 \AA$ for the $\mathrm{Si}-\mathrm{Si}$ bond, respectively; the latter appears too short as a consequence of charge fluctuations leading to an unduly polar bond. According to this result, we expect the adatom structure to begin to break up at a Ge coverage of approximately $0.4 \mathrm{ML}$, nearly equivalent to the saturation of all Si dangling bonds in the DAS model. This is consistent with the XSW results for higher coverages. ${ }^{30}$

In order to understand the formation of this stable adsorption conformation, we have calculated, as a first step, the adsorption of the third Ge on top of that Ge atom adsorbed on the Si adatom. Such an arrangement, however, turned out to be impossible, i.e., it did not lead to a stable geometry. To examine this process in more detail, possible reaction paths for the approach of an additional $\mathrm{Ge}$ atom have to be figured out when all $\mathrm{Si}$ adsorption sites are already occupied. To this end, further calculations are currently in progress to obtain the electrostatic potential in the vicinity of the Ge-deposited surface in order to determine such reaction paths.

\section{COMPARISON WITH EXPERIMENT}

Since no theoretical investigations of the Ge adsorption on $\mathrm{Si}(111)-7 \times 7$ surfaces have been published so far, we correlate our results only to the available experimental data. Gossmann et al. ${ }^{28,29}$ report that the $\mathrm{Si}(111)-7 \times 7$ superstructure transforms into a perfect $1 \times 1$ surface upon $\mathrm{Ge}$ deposition of $\Theta \approx 1.3 \mathrm{ML}$ at $T=570 \mathrm{~K}$ but that it does not take place at room temperature. This reconstruction is in accordance with our calculations insofar as the adsorption of a third Ge atom leads to a stable structure only if the adatom structure is broken up. However, our calculations correspond to Ge coverages of about 0.5 ML, and we did not follow the growth process in detail. Consequently, a Ge coverage of $\Theta=1.3 \mathrm{ML}$ is too high for an instructive comparison with our calculations.

Patel et al. ${ }^{31}$ deposited approximately $0.8 \mathrm{ML} \mathrm{Ge}$ on a Si(111) $-7 \times 7$ substrate at a rate of about $1 \mathrm{ML} / \mathrm{sec}$ which exceeds that of Gossmann et al. ${ }^{28}$ by a factor of 600 . Accordingly, the possibility cannot be excluded that they have measured growth processes perturbed by Ge-Ge collisions during the adsorption. This effect could be significant because Patel et al.$^{31}$ have kept the substrate at room temperature during epitaxy, thereby impeding the $\mathrm{Ge}$ atoms from finding their ideal adsorption site through surface diffusion. Furthermore, their interpretation is not based on an adatom model and assumes the upper two silicon surface layers are unrelaxed. Moreover, they did not work under ultrahigh vacuum (UHV) conditions and added an amorphous silicon cap of $100 \AA$ to protect the Ge layer. According to Gibson et al ${ }^{32}$ such a cap preserves the $7 \times 7$ superstructure but presumably removes the adatom layer. Yet, in the [111] direction Patel et al. ${ }^{31}$ observed a phase value of $1.02 d_{111}$ $\left(d_{111}=3.14 \AA\right)$ of the Fourier component of the Ge distribution function, close to the value of $1.06 d_{111}$ for $\Theta_{\mathrm{Ge}}=0.4 \mathrm{ML}$ measured by Dev et al. ${ }^{25,26}$

In a second paper, Patel et al. ${ }^{33}$ base the interpretation of their XSW measurements under UHV conditions on the DAS model but the Ge coverage of $\Theta \approx 1 \mathrm{ML}$ still does not allow any conclusion about the preferred adsorption site because an occupation of all six surface sites in a $7 \times 7$ unit cell corresponds to about $0.12 \mathrm{ML}$ only. In the [111] direction they measured a phase value of $1.04 d_{111}$ with a coherent fraction of 0.44 but they did not give any details about the Ge positions and coverage sites. Therefore, this paper does not contain any valuable information with regard to our calculations.

Dev et al. ${ }^{26}$ performed XSW measurements on the $\mathrm{Ge} / \mathrm{Si}(111)-7 \times 7$ interface under UHV conditions for $\mathrm{Ge}$ coverages of $0.2,0.4$, and $0.5 \mathrm{ML}$ at a substrate temperature of $530^{\circ} \mathrm{C}$. They interpret the coverage of $0.2 \mathrm{ML}$ in the frame of the DAS model as the Ge occupation of all six surface-atop sites, whereas the coverages of 0.4 and $0.5 \mathrm{ML}$ are interpreted as the occupation of all atop sites; the last value allows a small incoherent fraction from random positions. Thereby, Dev et al.$^{25,26}$ introduce three assumptions in their interpretation. First, they assume the height of the adatom over the first Si layer to be 0.78 $\AA$ based on the STM experiments of Binnig et al. ${ }^{21}$ Second, the $\mathrm{Si}-\mathrm{Ge}$ bond length is given the same value of $2.40 \AA$ for both adsorption sites, which is the arithmetic average of the $\mathrm{Si}$ and $\mathrm{Ge}$ bulk bond lengths. Finally, they choose the Si bilayer separation also to be $0.78 \AA$ corresponding to an unrelaxed geometrical configuration. These assumptions have to be revised according to our results: On the one hand, the calculated $\mathrm{Si}-\mathrm{Ge}$ bond lengths are substantially different for both adsorption sites. On the other hand, the adatom position deviates considerably from the assumed $1.56 \AA$ over the second 
layer. Remarkably, both errors almost cancel each other since, according to our calculations, the average $\mathrm{Si}-\mathrm{Ge}$ bond length with six $\mathrm{Ge}$ atoms adsorbed on surface-atop sites and 12 on adatom-atop ones, corresponding to a coverage of about $0.4 \mathrm{ML} \mathrm{Ge}$, is $2.38 \AA$ and thus close to $2.40 \AA$. For a Ge coverage of $0.2 \mathrm{ML}$ which equals an occupation of only the surface-atop sites, Dev et al. ${ }^{25}$ measure in the [111] direction the Ge distance from the (111) diffraction plane which is the middle of the Si bilayer in the absence of relaxation. The resulting $\mathrm{Si}-\mathrm{Ge}$ distance is $2.34 \pm 0.06 \AA$, a value being most likely too short. For Becker et al. ${ }^{34}$ observed a contraction by 0.2 $\AA$ with STM in the faulted subcell of the DAS model that would lead to a $\mathrm{Si}-\mathrm{Ge}$ bond length of $2.44 \AA$, significantly closer to our results. Independently of this reasoning, a mechanism producing the short $\mathrm{Si}-\mathrm{Ge}$ bond length in the XSW experiments of Dev et al. ${ }^{25}$ could be a possible contraction of the surface-atop site upon adsorption. Additionally, according to the recent calculation by Qian and Chadi ${ }^{35}$ more than just the upper two Si layers exhibit a relaxation, which seems more plausible than a relaxation of only the topmost two layers as reported by Takayanagi et al. ${ }^{2}$ In summing up, we can conclude from our results that the actual $\mathrm{Si}-\mathrm{Ge}$ bond length for direct surface-atop adsorption should range between 2.40 and $2.52 \AA$. Finally, taking the observations from Dev et al. ${ }^{25,26}$ for granted, the interpretation of the XSW measurements by Durbin et al. ${ }^{20}$ on the bare $\mathrm{Si}(111) 7 \times 7$ surface would require the unrealistic $\mathrm{Si}$ Ge distance of $2.90 \AA$, supporting our reasoning in Sec. III.

For $\Theta_{\mathrm{Ge}}=0.4 \mathrm{ML}$ Dev et al. ${ }^{25}$ interpret their experiment as a complete Ge occupation of surface-atop and adatom-atop sites, the latter being possible only after all surface sites are occupied. This agrees exactly with our calculations that adatom adsorption of $\mathrm{Ge}$ is possible but distinctly less favorable. Such an interpretation gets further support from their measurements for Ge coverages of 0.2 and $0.5 \mathrm{ML}$ in the [220] direction where the higher coverage corresponds to an occupation of all 18 adsorption sites in a $7 \times 7$ unit cell (or 19 including the corner hole). In this case, the observed Ge position was $0.75 d_{220}$ $\left(d_{220}=1.92 \AA\right)$ instead of $0.72 d_{220}$ as expected by them. This deviation is slightly above the given error bar of $0.01 d_{220}$, the reason being simply the erroneous assumption of the too short adatom height, which gains importance with higher coverages when more Ge atoms are adsorbed on adatoms than on surface-site atoms.

\section{CONCLUSIONS}

In this paper, we have presented the first theoretical results about $\mathrm{Ge}$ adsorption on a reconstructed $\mathrm{Si}(111)$ $7 \times 7$ surface by cluster model calculations. Ge adsorption on adatoms is found to be possible but energetically less favorable than direct surface-atop adsorption, in agreement with experimental data. Thus, adatom adsorption takes place only after all surface sites are occupied. Our calculated distances are consistent with the available experimental data leading, at the same time, to revised interpretations in some cases. For Ge coverages higher than about 0.4 ML no additional Ge adsorption is possible without substantial structural changes. Stable structures are obtained by the rearrangement of the adatom structure, equivalent to the experimentally observed transformation from a $7 \times 7$ superstructure into a $1 \times 1$ lattice. A theoretical explanation of the initial steps of $\mathrm{Ge} / \mathrm{Si}(111)-7 \times 7$ interface formation has been tried. In order to understand this process in more detail, the electrostatic potential of the substrate-adsorbate system is currently being computed from the charge density at the cluster surface and between the adsorbates.

\section{ACKNOWLEDGMENTS}

We wish to thank B. N. Dev and G. Materlik for helpful discussion on the experimental background and for reading the manuscript.
${ }^{*}$ Present address: University of New Orleans, Department of Chemistry, Lakefront, New Orleans, LA 70148.

$\dagger$ Present address: University of Illinois at Urbana-Champaign, Department of Physics, 1110 West Green Street, Urbana, IL 61801 .

${ }^{1}$ K. Takayanagi, Y. Tanishiro, S. Takahashi, and M. Takahashi, Surf. Sci. 164, 367 (1985).

${ }^{2}$ K. Takayanagi, Y. Tanishiro, S. Takahashi, and M. Takahashi, J. Vac. Sci. Technol. A 3, 1502 (1985).

${ }^{3}$ M. Grodzicki, J. Phys. B 13, 2683 (1980).

${ }^{4}$ O. Kühnholz, Diplom thesis, University of Hamburg, 1987.

${ }^{5}$ M. Grodzicki and O. Kühnholz, J. Mol. Struct. 174, 65 (1988).

${ }^{6}$ O. Zakharieva-Pencheva, H. Böse, H. Förster, W. Frede, and M. Grodzicki, J. Mol. Struct. 122, 101 (1985).

${ }^{7}$ H. Förster, M. Grodzicki, and O. Zakharieva-Pencheva, J. Mol. Struct. 143, 267 (1986).

${ }^{8}$ M. Grodzicki, H. Förster, R. Piffer, and O. ZakharievaPencheva, Catal. Today 3, 75 (1988).

${ }^{9} \mathrm{M}$. Hütsch, Diplom thesis, University of Hamburg, 1987.

${ }^{10} \mathrm{M}$. Hütsch and M. Grodzicki, in Lecture Notes in Physics, edited by E. R. Hilf, F. Kammer, and K. Wien (Springer, Berlin, 1987), Vol. 269, p. 212.

${ }^{11}$ K. Raghavachari, J. Chem. Phys. 84, 5672 (1986).

${ }^{12}$ G. Pacchioni and J. Koutecky, J. Chem. Phys. 84, 3301 (1986).

${ }^{13}$ T. P. Martin and H. Schaber, Z. Phys. B 35, 61 (1979).

${ }^{14} \mathrm{~K}$. Raghavachari and V. Logovinsky, Phys. Rev. Lett. 55, 2853 (1985).

${ }^{15}$ B. Kunz, in Theory of Chemisorption, edited by J. R. Smith (Springer, Berlin, 1980).

${ }^{16}$ G. X. Qian and D. J. Chadi, Phys. Rev. B 35, 1288 (1987).

${ }^{17}$ M. Grodzicki, V. Männing, A. X. Trautwein, and J. M. Friedt, J. Phys. B 20, 5595 (1987).

${ }^{18}$ J. E. Northrup, Phys. Rev. Lett. 57, 154 (1986).

${ }^{19}$ W. Daum, H. Ibach, and J. E. Müller, Phys. Rev. Lett. 59, 1593 (1987).

${ }^{20}$ S. M. Durbin, L. E. Berman, and B. W. Batterman, Phys. Rev. Lett. 56, 236 (1986).

${ }^{21}$ G. Binnig, H. Rohrer, Ch. Gerber, and E. Weibel, Phys. Rev. Lett. 50, 120 (1983).

${ }^{22}$ R. J. Hamers, R. M. Tromp, and J. E. Demuth, Phys. Rev. 
Lett. 56, 1972 (1986).

${ }^{23}$ R. P. Messmer, in The Nature of the Surface Chemical Bond, edited by T. N. Rhodin and G. Ertl (North-Holland, Amsterdam, 1979).

${ }^{24}$ J. E. Demuth, B. N. J. Persson, and A. J. Schell-Sorokin, Phys. Rev. Lett. 51, 2214 (1983).

${ }^{25}$ B. N. Dev, G. Materlik, F. Grey, R. L. Johnson, and M. Clausnitzer, Phys. Rev. Lett. 57, 3058 (1986).

${ }^{26}$ B. N. Dev, G. Materlik, R. L. Johnson, W. Kranz, and P. Funke, Surf. Sci. 178, 1 (1986).

${ }^{27}$ O. Gunnarsson and R. O. Jones, Phys. Rev. B 31, 7588 (1985).

${ }^{28}$ H.-J. Gossmann, L. C. Feldman, and W. M. Gibson, Phys. Rev. Lett. 53, 294 (1984).

${ }^{29}$ H.-J. Gossmann, L. C. Feldman, and W. M. Gibson, Surf. Sci.
155, 413 (1985).

${ }^{30}$ B. N. Dev, G. Materlik, F. Grey, and R. L. Johnson (unpublished).

${ }^{31}$ J. R. Patel, J. A. Golovchenko, J. C. Bean, and R. J. Morris, Phys. Rev. B 31, 6884 (1985).

${ }^{32}$ J. M. Gibson, H.-J. Gossmann, J. C. Bean, R. T. Tung, and L. C. Feldman, Phys. Rev. Lett. 56, 355 (1986).

${ }^{33}$ J. R. Patel, P. E. Freeland, J. A. Golovchenko, A. R. Kortan, D. J. Chadi, and G. X. Qian, Phys. Rev. Lett. 57, 3077 (1986).

${ }^{34}$ R. S. Becker, J. A. Golovchenko, E. G. McRae, and B. S. Swartzentruber, Phys. Rev. Lett. 55, 2028 (1985).

${ }^{35}$ G. X. Qian and D. J. Chadi, J. Vac. Sci. Technol. B 4, 1079 (1986). 